\title{
Performance visual após implante de LIO tórica em pacientes com astigmatismo corneano
}

\author{
Visual performance after toric IOL implantation in patients \\ with corneal astigmatism
}

\author{
Renato José Bett Correia ${ }^{1}$ \\ Hamilton Moreira² \\ Sólon Uchoa Lagó Netto ${ }^{3}$ \\ Gleisson Rezende Pantaleão ${ }^{4}$
}

Trabalho realizado no Hospital de Olhos do Paraná Curitiba (PR) - Brasil.

${ }^{1}$ Fellow de Segmento Anterior pelo Hospital de Olhos do Paraná - Curitiba (PR) - Brasil.

Doutor, Professor Adjunto da Universidade Federal do

Paraná; Professor Assistente da Faculdade Evangélica do Paraná - Curitiba (PR) - Brasil.

${ }^{3}$ Fellow de Segmento Anterior pelo Hospital de Olhos do Paraná - Curitiba (PR) - Brasil.

${ }^{4}$ Fellow de Segmento Anterior pelo Hospital de Olhos do Paraná - Curitiba (PR) - Brasil.

Endereço para correspondência: Hamilton Moreira. Rua Coronel Dulcídio, 199 - 4ªndar - Curitiba (PR) CEP 80420-170

E-mail: hamiltonmoreira@onda.com.br

Recebido para publicação em 05.08.2008

Última versão recebida em 09.07.2009

Aprovação em 14.07.2009

\section{RESUMO}

Objetivo: Avaliar acuidade visual final sem correção e com correção e estabilidade rotacional da LIO. Métodos: Estudo prospectivo de 20 olhos de 13 pacientes submetidos à facoemulsificação, por catarata associado a astigmatismo ceratométrico regular, simétrico, variando de 1 a 4 dioptrias. No pré-operatório foram avaliadas a melhor acuidade visual corrigida, a refração, a ceratometria e topografia computadorizada. O cálculo do poder cilíndrico da lente e seu posicionamento foram determinados pelo fabricante. Todas as lentes foram implantadas por um mesmo cirurgião, dentro do saco capsular. Os pacientes foram analisados por um segundo observador, no $1^{\circ}, 10^{\circ}, 20^{\circ}, 30^{\circ}$ e $60^{\circ}$ dia pós-operatório. Resultados: A acuidade visual dos pacientes deste estudo sem correção variou entre 20/15 e 20/40, sendo 1 olho com 20/15 (5\%), 4 olhos 20/20 (20\%), 6 olhos 20/25 (30\%), 7 olhos 20/30 (35\%) e 2 olhos 20/40 (10\%). A acuidade visual com a melhor correção variou entre $20 / 15$ e 20/40, sendo 2 olhos com 20/15(10\%), 9 olhos 20/20 (45\%), 7 olhos 20/25 (35\%), 1 olho 20/30(5\%) e 1 olho 20/40 (5\%). Lembrando ainda que a refração esférica média foi de $-0,05 \mathrm{DE}$ variando entre $-0,50$ até $+0,75$. Refração cilíndrica teve média de $-0,63$ DC, variando entre $-0,50$ e $-1,25$ DC. A rotação da LIO teve uma média de $3,2^{\circ}$ até o $30^{\circ}$ dia pós-operatório, variando de $0^{\circ}$ de rotação e rotação máxima de $13^{\circ}$, sendo que 7 lentes (35\%) não sofreram rotação, 9 lentes $(45 \%)$ sofreram rotação entre $1^{\circ}$ e $5^{\circ}, 3$ lentes $(15 \%)$ tiveram rotação entre $6^{\circ}$ e $10^{\circ}$ e por fim 1 lente $(5 \%)$ teve rotação entre $11^{\circ}$ e $15^{\circ}$. Não houve rotação significativa após o $30^{\circ}$ dia pós-operatório. Discussão: A média da rotação da LIO foi de $3,2^{\circ}$, onde $95 \%$ das LIO tiveram rotação menor ou igual a $10^{\circ}$, o que se traduz em uma estabilidade rotacional muito boa. Na prática percebemos que a boa acuidade visual está diretamente relacionado à sua estabilidade rotacional e previsibilidade refracional.

Descritores: Astigmatismo/cirurgia; Implante de lente intraocular/métodos; Facoemulsificação; Extração de catarata; Acuidade visual; Complicações pós-operatórias

\section{INTRODUÇÃO}

$\mathrm{O}$ astigmatismo corneano tem sido fator limitante da cirurgia de catarata por um longo tempo ${ }^{(1)}$, hoje existem várias técnicas disponíveis para o seu tratamento. Terapias estas que se estendem desde simples óculos com lentes de poder cilíndrico, lentes de contato rígidas e gelatinosas até um extenso número de técnicas cirúrgicas como incisões relaxantes corneanas $^{(2-3)}$ e limbares ${ }^{(4)}$, posição seletiva da incisão na facoemulsificação $o^{(5)}$, 
procedimentos fotorrefrativos a laser ${ }^{(6)}$, implantes intraestromais $^{(7)}$ e lentes intraoculares tóricas ${ }^{(8)}$.

As lentes intraoculares tóricas foram inicialmente usadas nas cirurgias de cataratas em 1994 por Shimizu et al. ${ }^{(9)}$, nessa ocasião eram lentes rígidas de três peças as quais necessitavam de sutura após cirurgia. Logo após, Grabow et al. obtiveram bons resultados com implante de LIO tórica dobrável de silicone $^{(10-11)}$

As lentes intraoculares tóricas têm sido alvo de estudo na ultima década como alternativa para correção de astigmatismo corneano em pacientes que já apresentam distúrbios visuais causados por opacificação cristaliniana ${ }^{(12)}$. As LIOs tóricas resultam em sucesso visual quando seu cilindro está em perfeito alinhamento com eixo do astigmatismo ${ }^{(4-5,13-14)}$. Sendo então a grande limitação dos bons resultados pós-operatórios, a rotação da $\mathrm{LIO}^{(4-5,13-15)}$.

\section{OBJETIVOS}

Avaliar a performance visual dos olhos dos pacientes que foram submetidos à cirurgia de extração do cristalino com implante de lente intraocular tórica. No pós-operatório foram avaliados a acuidade visual sem e com correção, além do componente esférico e cilíndrico da refração e analisada a rotação pós-operatória da LIO.

\section{MÉTODOS}

Este é um ensaio clínico, prospectivo, não controlado, que incluiu 20 olhos de 13 pacientes, dos quais todos apresentavam catarata associada à baixa acuidade visual, com astigmatismo ceratométrico regular, simétrico, com eixos ortogonais, variando entre 1 a 4 dioptrias cilíndricas. O componente esférico da lente foi compatível com as dioptrias disponíveis pelo fabricante.

Os critérios de inclusão foram presença de catarata uni ou bilateral, em pacientes acima de 18 anos, com capacidade de compreensão para assinar termo de consentimento e ausência de comorbidades que comprometessem a acuidade visual final. Foram excluídas complicações cirúrgicas relacionadas ao saco capsular (deiscência de zônula, comprometimento da capsulorrexis), endoftalmite e falta do seguimento do paciente.

Todos os pacientes inseridos no estudo assinaram o termo de consentimento livre e esclarecido. Não houve paciente que se recusou a ingressar no presente estudo ou a assinar o termo. $\mathrm{O}$ projeto do estudo foi submetido à apreciação e aprovado pelo Comitê de Ética em Pesquisa da Sociedade Evangélica Beneficente de Curitiba, protocolado sob o número 4420/08.

No pré-operatório foi avaliada a melhor acuidade visual corrigida dos pacientes, assim como a refração usada, ceratometria e topografia computadorizada baseada nos anéis de Plácido (Eye Sys Vista ${ }^{\circledR}$. Texas, USA). O comprimento axial foi determinado com ultrassonografia de imersão AScan (Ocuscan XP, Alcon ${ }^{\circledR}$ ). O cálculo do poder esférico da
LIO foi determinado considerando o comprimento axial dos olhos estudados, sendo usada a formula Holladay para comprimentos axiais variando entre 22 a $24 \mathrm{~mm}$, a regra SRK-T foi utilizada nos olhos com mais de $24 \mathrm{~mm}$ e Hoffer Q usada nos olhos que apresentaram comprimento menor que $22 \mathrm{~mm}$, objetivando a emetropia. O cálculo do poder cilíndrico da lente e seu posicionamento foram determinados pelo seu fabricante levando em consideração as ceratometrias maior e menor, poder esférico da LIO, posição da incisão e astigmatismo induzido pelo cirurgião. Este cálculo é realizado pelo fabricante da LIO (Alcon ${ }^{\circledR}$ ) através do site www.acrysoftoriccalculator.com

A lente intraocular foi a SN60T AcrySof ${ }^{\circledR}$ Toric $^{\circledR}$ - Alcon ${ }^{\circledR}$, sendo ela acrílica, hidrofóbica, com plataforma de peça única, com constante A de 118,4 para biometria por ultrassom e 118,9 para biometria óptica, com marcas (três pontos alinhados) nos eixos onde se localiza o poder cilíndrico da lente o qual serve de referência para o preciso posicionamento da lente. Essas lentes possuem três apresentações de poder cilíndrico, são elas T3 (1,5 DC), T4 (2,25 DC) e T5 (3,0 DC).

Com o paciente sentado, sob anestesia tópica (proximetacaína), olhando para o infinito em um eixo horizontal, foram feitas duas marcas no limbo, a $0^{\circ} \mathrm{e} 180^{\circ} \mathrm{com}$ marcador pendular. Após a marcação, os pacientes foram submetidos a bloqueio peribulbar. Durante o ato operatório, foi realizada a marcação da incisão principal a $135^{\circ}$ e a marcação do eixo para orientar o posicionamento da LIO com auxílio de transferidor cirúrgico. Realizada incisão corneana de $2,75 \mathrm{~mm}$ no eixo de $135^{\circ}$, capsulorrexis circular contínua. Todos os 20 olhos foram submetidos à facoemulsificação pela técnica cirúrgica "stop and chop". Todas as lentes foram implantadas dentro do saco capsular. A substância viscoelástica foi retirada e após posicionamento das marcações da LIO foi realizada hidratação das incisões cirúrgicas.

Os pacientes foram analisados por um segundo observador independente, mascarado, em lâmpada de fenda, no $1^{\circ}, 10^{\circ}$, $20^{\circ}, 30^{\circ}$ e $60^{\circ}$ dia pós-operatório. Avaliou-se acuidade visual sem e com correção, a refração, a ceratometria, topografia computadorizada, o exame de frente de ondas (Ladar Wave ${ }^{\circledR}$ da Alcon ${ }^{\circledR}$ ) e análise da rotação da LIO sob dilatação seguindo a mesma metodologia da marcação do eixo pré-operatória, onde com o paciente sentado, sob anestesia tópica (proximetacaína) e pupilas dilatadas, olhando para o infinito em um eixo horizontal, são realizadas duas marcas no limbo, a $0^{\circ}$ e $180^{\circ} \mathrm{com}$ marcador pendular. Após a marcação, os pacientes são examinados em lâmpada de fenda e com ajuda de um transferidor avaliamos a posição das marcas da LIO e comparamos com o eixo que foi programado na cirurgia.

\section{RESULTADOS}

Foram avaliados 20 olhos de 13 pacientes com média de idade de 75,69 anos, variando entre 58 e 88 anos, sendo realizado o procedimento cirúrgico em 8 mulheres $(61,5 \%)$ e 5 homens $(38,5 \%)$. 


\section{Resultado visual sem correção}

A acuidade visual dos pacientes deste estudo sem correção variou entre 20/15 e 20/40 sendo: 1 olho com 20/15 (5\%), 4 olhos 20/20 (20\%), 6 olhos 20/25 (30\%), 7 olhos 20/30 (35\%) e 2 olhos 20/40 (10\%).

\section{Resultado visual com melhor correção}

A acuidade visual com melhor correção variou entre 20/15 e 20/40 sendo: 2 olhos com 20/15 (10\%), 9 olhos 20/20 (45\%), 7 olhos 20/25 (35\%), 1 olho 20/30 (5\%) e 1 olho 20/40 (5\%) (Gráfico 1).

\section{Resultado refracional}

A refração esférica média foi de $-0,05 \mathrm{DE}$ variando entre $-0,50$ até $+0,75 \mathrm{DE}$. Refração cilíndrica teve média de $-0,63 \mathrm{DC}$, variando entre $-0,50$ e $-1,25 \mathrm{DC}$.

\section{Rotação pós-operatória da LIO}

A rotação da LIO nesse estudo teve uma média de $3,2^{\circ}$ até o $30^{\circ}$ dia pós-operatório, variando de $0^{\circ}$ de rotação e rotação máxima de $13^{\circ}$, sendo que 7 lentes (35\%) não sofreram rotação, 9 lentes $(45 \%)$ sofreram rotação entre $1^{\circ}-5^{\circ}, 3$ lentes (15\%) tiveram rotação entre $6^{\circ}$ e $10^{\circ}$ e 1 lente $(5 \%)$ teve rotação entre $11^{\circ}$ e $15^{\circ}$. Não houve rotação significativa após o $30^{\circ}$ dia pósoperatório. A tabela 1 correlaciona o grau de rotação da LIO com a melhor acuidade visual corrigida gerada no paciente. E o gráfico 2 mostra a significância estatística desta correlação com $\mathrm{p}=0,023$.

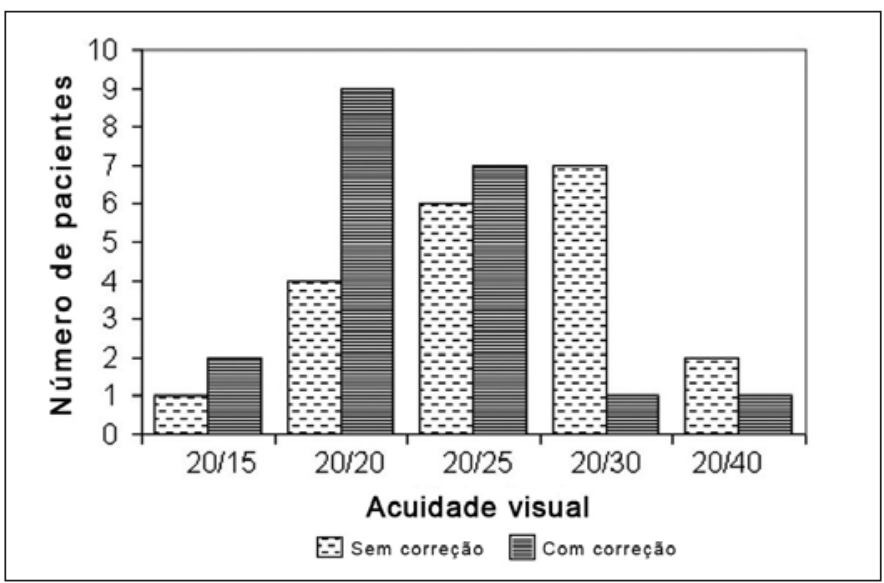

Gráfico 1 - Comparação da acuidade visual não corrigida e corrigida dos pacientes com lente intraocular implantada

Tabela 1. Correlação direta entre rotação da lente e acuidade
visual
\begin{tabular}{lllll} 
AV / Rotação & $0^{\circ}$ & $\mathbf{1}^{\circ}-5^{\circ}$ & $\mathbf{6}^{\circ}-\mathbf{1 0}^{\circ}$ & $\mathbf{1 1}^{\circ}-\mathbf{1 5}^{\circ}$ \\
$20 / 15$ & $1(5 \%)$ & & & \\
$20 / 20$ & $3(15 \%)$ & $3(15 \%)$ & $1(5 \%)$ & \\
$20 / 25$ & $2(10 \%)$ & $3(15 \%)$ & $1(5 \%)$ & \\
$20 / 30$ & $1(5 \%)$ & $2(10 \%)$ & & $1(5 \%)$ \\
$20 / 40$ & & $1(5 \%)$ & $1(5 \%)$ & \\
\hline
\end{tabular}

\section{Aberrações pós-operatórias}

Os pacientes deste estudo foram submetidos ao exame de aberrometria com exame de frente de ondas onde foi principalmente observado o astigmatismo residual, Defocus, Coma e aberração esférica. $\mathrm{O}$ astigmatismo residual observado no pósoperatório através de exame de frente de onda teve média de $-0,81$ DC variando entre - 0,26 e - 1,63 DC. O Defocus observado teve valor médio de 2,20 variando de 0,84 até 5,45 . O Coma observado teve índice médio de 0,28 variando entre 0,09 até 0,62 . E a aberração esférica com índice médio de 0,51 variando entre 0,20 até 1,02. O teste de Correlação de Pearson mostrou que a rotação da lente aumenta as aberrações de maneira estatisticamente significativa, sendo no Defocus $\mathrm{p}=0,061$, o Coma com $\mathrm{p}=0,510$ e aberração esférica (Spherical Aberration) com $\mathrm{p}=0,025$. Correlações estas mostradas nos gráficos de 3 e 4 .

\section{Complicações}

Não houve intercorrências per-operatórias e pós-operatória. Lembrando ainda que nenhum paciente deste grupo apresentou comorbidade ocular ou sistêmica.

\section{DISCUSS ÃO}

O nosso estudo mostrou que a média da rotação da LIO foi de $3,2^{\circ}$, onde $95 \%$ das LIOs tiveram rotação menor ou igual a $10^{\circ}$, o que se traduz em uma estabilidade rotacional muito boa se compararmos com estudos como o de Shimizu et al. ${ }^{(9)}$ ou Ruhswurm et al. ${ }^{(16)}$, os quais obtiveram rotações de até $30^{\circ} \mathrm{com}$ suas médias girando em torno de $15^{\circ}$ de rotação, em nosso trabalho a rotação máxima foi de $13^{\circ}$. Trabalhos mais modernos como o de Till et al. ${ }^{(17)}$ mostram que $11 \%$ dos pacientes apresentaram rotação da LIO de até $15^{\circ}$, contra $5 \%$ observado no presente estudo.

A performance visual da lente pode ser considerada ótima, uma vez que em 20 olhos temos 18 (90\%) com visão de

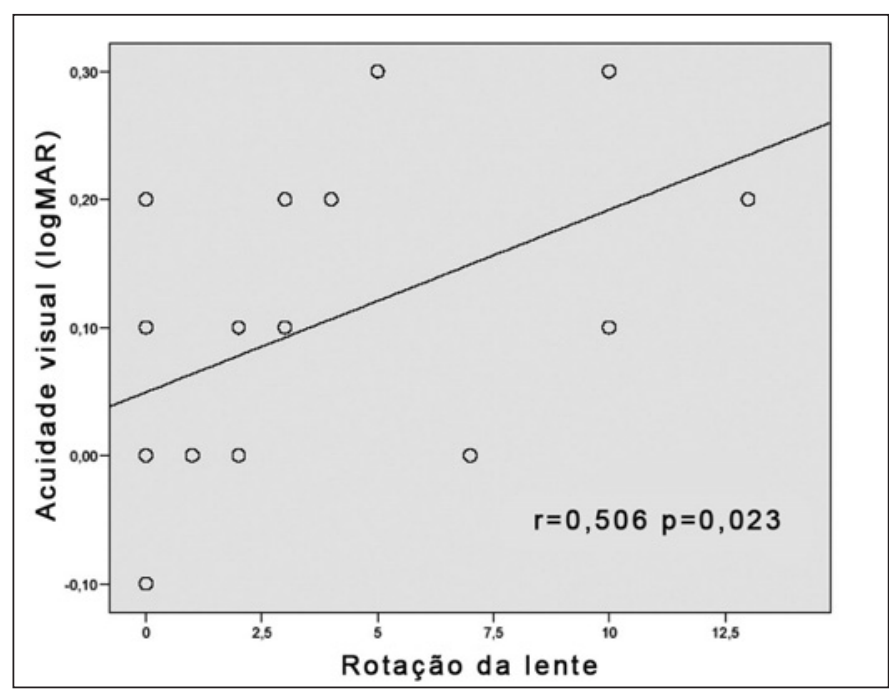

Gráfico 2 - Relação estatística entre rotação da lente e acuidade visual, calculada com teste de correlação de Pearson 


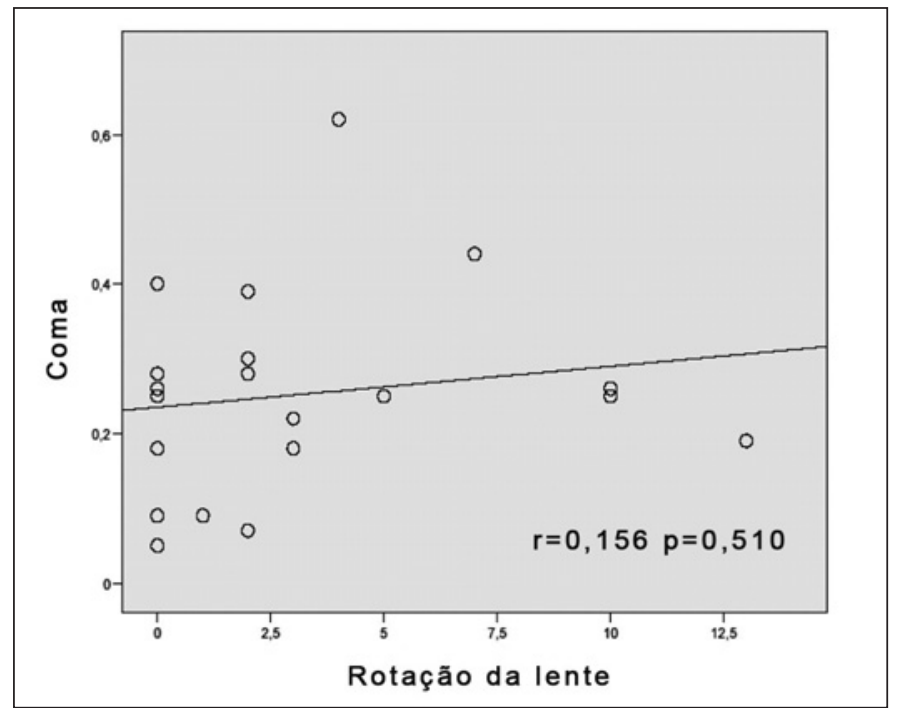

Gráfico 3 - Análise estatística entre Coma e rotação da lente

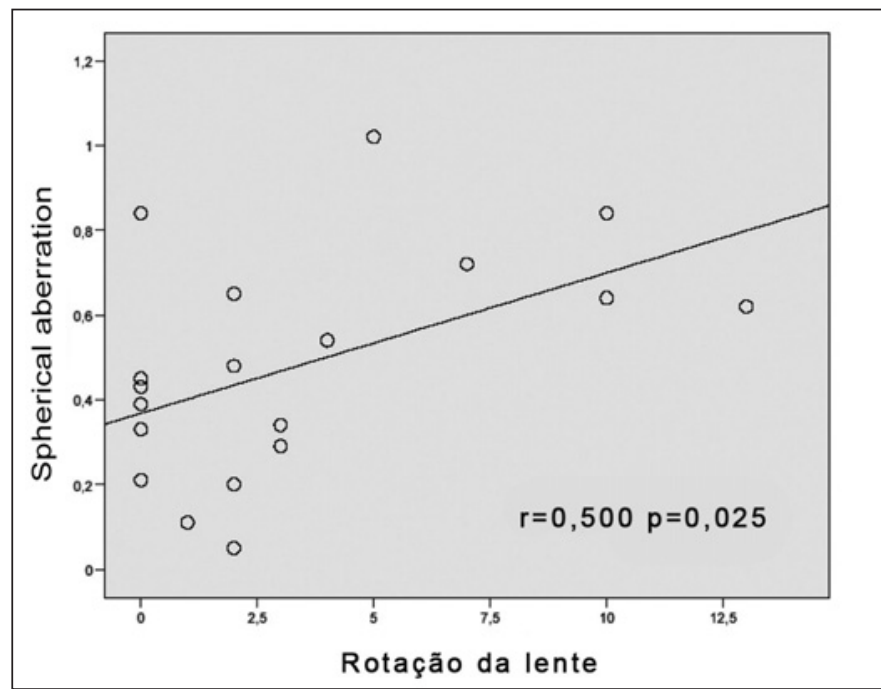

Gráfico 4 - Correlação estatística entre rotação da lente e aberração esférica (Spherical Aberration)

20/25, ou melhor, com correção óptica e 11 (55\%) com visão de 20/25 ou melhor sem correção; e isto mostra também que a previsibilidade refracional também foi ótima, onde apenas 9 olhos necessitaram efetivamente de correção para atingir uma melhor visão sendo que o resultado refracional foi baixo, com uma média de -0,05 DE e -0,63 DC.

$\mathrm{Na}$ prática percebemos que uma rotação de $1^{\circ}$ da lente pode causar perda de 3,3\% do poder cilindro da LIO sobre o astigmatismo corneano e quando essa rotação é próxima de $30^{\circ}$ anula-se o poder refrativo cilíndrico da $\mathrm{LIO}^{(18)}$. Este estudo mostrou ainda haver correlação estatisticamente significativa entre a rotação da lente e diminuição da acuidade visual, assim como a correlação da rotação da lente e aumento das aberrações. Isso nos mostra que o sucesso da LIO está diretamente relacionado com sua estabilidade rotacional.
Conclui-se que o implante deste modelo de lente intraocular tórica tem uma grande estabilidade rotacional, com uma média de rotação pós-operatória baixa, oferecendo ao paciente excelente performance visual como mostrado pelas estatísticas no exame de aberrometria.

\section{ABSTRACT}

Purpose: To analyze visual acuity without correction and rotational stability outcomes following toric IOL implantation. Methods: Prospective study of 20 eyes of 13 patients that underwent phacoemulsification surgery indicated for cataract associated with regular keratomeric astigmatism, symmetrical, ranging from 1 to 4 diopters. Best corrected visual acuity, refraction, keratometry and computed topography were performed preoperatively. The calculation of cylindrical lens power and its placement were determined by the manufacturer. All lenses were implanted in the capsular bag by the same surgeon. The patients were examined by a second independent observer, at $1^{\text {st }}, 10^{\text {th }}, 20^{\text {th }}, 30^{\text {th }}$, and $60^{\text {th }}$ postoperative day. Results: Visual acuity without correction ranged between $20 / 15$ and 20/40. One eye achieved 20/15 (5\%), 4 eyes 20/20 (20\%), 6 eyes 20/25 (30\%), 7 eyes 20/30 (35\%) and 2 eyes 20/40 (10\%). Best corrected visual acuity ranged between 20/15 and 20/40; two eyes with 20/15 (10\%), 9 eyes 20/20 (45\%), 7 eyes 20/25 (35\%), 1 eye 20/30 (5\%) and 1 eye 20/40 (5\%). It is important to remember that the average spherical refraction was $-0.05 \mathrm{SD}$ (ranging from -0.50 to $+0.75 \mathrm{SD}$ ). The mean cylindrical refraction was $-0.63 \mathrm{CD}$ ranging from -0.50 to $-1.25 \mathrm{CD}$. The IOL rotation in this study had an average of $3.2^{\circ}$ to $30^{\circ}$, ranging from $0^{\circ}$ of rotation to a maximum of $13^{\circ}$; 7 lenses $(35 \%)$ suffered no rotation, 9 lenses $(45 \%)$ suffered rotation between $1^{\circ}$ to $5^{\circ}, 3$ lenses $(15 \%)$ had rotation between $6^{\circ}$ to $10^{\circ}$, and ultimately 1 lens (5\%) had rotation between $11^{\circ}$ to $15^{\circ}$. There was no significant rotation after the $30^{\text {th }}$ postoperative day. Discussion: The average of rotation of the IOL was $3.2^{\circ}$, where $95 \%$ of IOLs presented rotation less than or equal to $10^{\circ}$ what means a very good rotational stability. In daily practice, a good visual acuity is directly related to IOL rotational stability and refractive predictability.

Keywords: Astigmatism/surgery; Lens implantation, intraocular/methods; Phacoemulsification; Cataract extraction; Visual acuity; Postoperative complications

\section{REFERÊNCIAS}

1. Sanders DR, Schneider D, Martin R, Brown D, Dulaney D, Vukich J, et al. Toric Implantable Collamer Lens for moderate to high myopic astigmatism. Ophthalmology. 2007;114(1):54-61.

2. Grabow HB. Intraocular correction of refractive errors. In: Kershner RM, editor. Refractive keratotomy for cataract surgery and the correction of astigmatism. Thorofare, NJ: Slack; 1994. p.79-115.

3. Müller-Jensen K, Fischer P, Siepe U. Limbal relaxing incisions to correct astigmatism in clear corneal cataract surgery. J Refract Surg. 1999;15(5):586-9. 
4. Tehrani M, Dick HB, Schwenn O, Blom E, Schmidt AH, Koch HR. Postoperative astigmatism an rotational stability after Artisan toric phakic intraocular lens implantation. J Cataract Refract Surg. 2003;29(9):1761-6.

5. Tehrani M, Dick HB. Incisional keratotomy to toric intraocular lenses: an overview of the correction of astigmatism in cataract and refractive surgery. Int Ophthalmol Clin. 2003;43(3):43-52.

6. Rao SN, Raviv T, Majmudar PA, Epstein RJ. Role of Orbscan II in screening keratoconus suspects before refractive corneal surgery. Ophthalmology. 2002; 109(9):1642-6

7. Oliveira CS, Moreira H, Wahab SA, Godoy G. Análise de nova técnica para o implante do anel de Ferrara no ceratocone. Arq Bras Oftalmol. 2004;67(3): 509-17.

8. Kersey JP, O'Donnell A, Illingworth CD. Cataract surgery with toric intraocular lenses can optimize uncorrected postoperative visual acuity in patients with marker corneal astigmatism. Cornea. 2007;26(2):133-5.

9. Shimizu K, Misawa A, Suzuki Y. Toric intraocular lenses: correcting astigmatism while controlling axis shift. J Cataract Refract Surg. 1994;20(5):523-6.

10. Grabow HB. Early results with foldable toric IOL implantation. Eur J Implant Refract Surg. 1994;6:177-8.

11. Grabow HB. Toric intraocular lens report. Ann Ophthalmol. 1997;29(3):161-3.
12. McMullan TF, Goldsmith C, Illingworth CD. Toric posterior chamber (in-thebag) intraocular lens implantation to correct postpenetrating keratoplasty astigmatism. Eye. 2007;21(1):150-2.

13. Tehrani M, Stoffelns B, Dick HB. Implantation of a custom intraocular lens with a 30-diopter torus for the correction of high astigmatism after penetrating keratoplasty. J Cataract Refract Surg. 2003;29(12):2444-7.

14. Gerten G, Michels A, Olmes A. Torische Intraokularlinsen. Klinische Ergebnisse und Rotationsstabilität. Ophthalmologe. 2001;98(8):715-20. Erratum in: Ophthalmologe. 2001;98(10):954.

15. Patel CK, Ormonde S, Rosen PH, Bron AJ. Postoperative intraocular lens rotation: a randomized comparison of plate and loop haptic implants. Ophthalmology. 1999;106(11):2190-5; discussion 2196.

16. Ruhswurm I, Scholz U, Zehetmayer M, Hanselmayer G, Vass C, Skorpik C. Astigmatism correction with a foldable toric intraocular lens in cataract patients. J Cataract Refract Surg. 2000;26(7):1022-7.

17. Till JS, Yoder PR Jr, Wilcox TK, Spielman JL. Toric intraocular lens implantation: 100 consecutive cases. J Cataract Refract Surg. 2002;28(2):295-301.

18. Linnola RJ, Sund M, Ylönen R, Pihlajaniemi T. Adhesion of soluble fibronectin laminin, and collagen type IV to intraocular lens materials. J Cataract Refract Surg. 1999;25(11):1486-91. 